\title{
FORUM
}

\section{Europäische Nachbarschaftspolitik nach der deutschen EU-Ratspräsidentschaft - Bilanz und Ausblick}

\author{
Günter Gloser*
}

Die erst 2003 ins Leben gerufene Europäische Nachbarschaftspolitik (ENP) hat sich in kürzester Zeit zu einem Grundpfeiler in den Beziehungen der Europäische Union zu ihren Nachbarländern entwickelt. Die ENP leistet einen wichtigen Beitrag zur Förderung von Stabilität, Sicherheit und Wohlstand in den Nachbarstaaten selbst und damit unmittelbar auch in der Europäischen Union. Eine stärkere Verflechtung mit der Europäischen Union, ebenso wie der ENP-Partner untereinander, trägt ganz wesentlich zu einer stabilen Sicherheitsarchitektur Europas bei. Sie zielt auf einen gemeinsamen Raum der Partnerschaft, Stabilität und der Solidarität mit unseren Nachbarn im Osten wie im Süden. Hierüber sind sich alle Mitgliedstaaten einig.

Obgleich die ENP im Kontext der letzten Erweiterungsrunden entstanden ist, greift sie in keiner Weise den künftigen Beziehungen zu den ENP-Partnerländern vor. Sie stellt keine Vorstufe zu einem EU-Beitritt dar, schlägt aber auch keine, Türen` zu. Die ENP ist dazu gedacht, neue Trennlinien zwischen der Europäischen Union und ihren Nachbarn zu vermeiden und stattdessen eine engere Vernetzung in allen Bereichen herbeizuführen. Hierzu gehört insbesondere eine wirtschaftliche Integration und eine sektorale Zusammenarbeit, aber auch engere politische und kulturelle Beziehungen. Die ENP ist ein einheitliches, kohärentes Instrument für den Süden und für den Osten. Mit Hilfe von individuell ausgehandelten Aktionsplänen gelingt es, die Heterogenität der verschiedenen ENP-Partner zu berücksichtigen und damit die notwendige Flexibilität herzustellen, um auf die unterschiedlichen Belange unserer Nachbarländer einzugehen.

\section{Die Stärkung der Europäischen Nachbarschaftspolitik unter der deutschen Präsi- dentschaft}

Aktionspläne für eine erste Gruppe von Nachbarn (Ukraine, Moldau, Tunesien, Marokko, Israel, Jordanien, Palästinensische Gebiete) wurden bereits Ende 2004 verabschiedet. Ihre Umsetzung hat begonnen und wurde im Dezember 2006 von der Kommission im Rahmen von Fortschrittsberichten evaluiert. Im Vorfeld der deutschen Präsidentschaft konnten weitere detaillierte Aktionspläne mit den südkaukasischen Ländern Armenien, Aserbaidschan, Georgien und zuletzt, Anfang 2007, mit dem Libanon und mit Ägypten vereinbart werden. Damit bestehen nun für zwölf der 16 ENP-Partnerländer entsprechende Aktionspläne.

Vor Beginn der deutschen Präsidentschaft wurden zudem die Finanzierungsinstrumente grundlegend reformiert. Hierbei spielt das Europäische Nachbarschafts- und Partnerschaftsinstrument (ENPI), das die bisherigen EU-Programme TACIS für den Osten (ohne Zentral-

Günter Gloser MdB, Staatsminister für Europa, Auswärtiges Amt. 
asien) und MEDA für den Süden ersetzt hat, eine zentrale Rolle. ${ }^{1}$ Vorrangig sollen die im Rahmen der Aktionspläne der Europäischen Nachbarschaftspolitik vereinbarten Schwerpunkte gefördert werden. Für die Mittelallokation ist neben den Bedürfnissen und der Absorptionsfähigkeit der Partnerländer insbesondere die Umsetzung der vereinbarten Reformen maßgeblich. Insgesamt sind circa 11,2 Milliarden Euro für den Zeitraum 2007-2013 veranschlagt.

Für eine Verstetigung und zur verstärkten Unterstützung dieser Reformprozesse in den ENP-Partnerländern, aber auch zur Betonung des Gestaltungswillens der Europäischen Union hatte sich die deutsche EU-Ratspräsidentschaft zum Ziel gesetzt, die ENP weiterzuentwickeln und zu vertiefen. Aufbauend auf die Mitteilung der Kommission vom 4. Dezember $2006^{2}$ wurde der deutschen EU-Ratspräsidentschaft ein Mandat erteilt, die ENP zu stärken und dem Europäischen Rat im Juni 2007 hierüber zu berichten. Die Mitgliedstaaten der Europäischen Union äußerten beim Rat für Allgemeine Angelegenheiten und Außenbeziehungen (RAA) vom 22. Januar ihre Unterstützung für eine intensivierte ENP. Erste konkrete Maßnahmen wurden umgesetzt. Hieran anknüpfend erstellte die Präsidentschaft sodann einen Fortschrittsbericht für den Europäischen Rat. Dieser Fortschrittsbericht enthält wichtige Bausteine für eine weitere Vertiefung der ENP. Er wurde durch den RAA am 18./19. Juni und den Europäischen Rat am 21. Juni indossiert.

\section{Grundprinzipien der ENP}

In den Schlussfolgerungen des RAA sowie insbesondere in dem Fortschrittsbericht werden nochmals die strategischen Ziele und Grundsätze der ENP betont. Die ENP ist in der EU-Außenpolitik von zentraler Bedeutung. Unsere Sicherheit und unser Wohlstand hängt maßgeblich davon ab, dass es uns gelingt, gemeinsam mit einem festen Kreis befreundeter Staaten, die gewaltigen Herausforderungen unserer Zeit, zum Beispiel in den Bereichen Energiesicherheit, Umwelt und Migration, zu bewältigen.

Die ENP ist eine umfassende Politik gegenüber unseren Nachbarn. Sämtliche Bereiche der EU-Beziehungen zum Partnerland werden hiervon erfasst. So enthalten auch die jeweiligen Aktionspläne detaillierte Vereinbarungen zu der gesamten Bandbreite der Zusammenarbeit.

Die ENP verfolgt einen leistungsbezogenen und kohärenten Ansatz. Sie richtet sich an alle Partner im Süden und im Osten. Ein ausgewogenes Verhältnis zwischen diesen beiden Regionen muss gewährleistet sein. Ein interregionaler Wettbewerb oder gar starre Allokationsquoten sollten vermieden werden. Jedes Partnerland wird nach Maßgabe der erreichten Fortschritte gefördert. Mit einer solchen leistungsbezogenen Differenzierung wird eine maßgeschneiderte Unterstützung der Partnerländer sichergestellt.

\section{Elemente einer vertieften ENP}

Die seit Einführung der ENP geschaffenen Anreize und die geleistete Unterstützung werden durch eine weitreichende wirtschaftliche Integration sowie eine verstärkte Zusammenarbeit bei sektorübergreifenden Themen noch verstärkt. Darüber hinaus fördert die ENP eine Vertiefung der regionalen Kooperation mit und insbesondere auch zwischen den ENP-Part-

1 Vgl. hierzu Verordnung (EG) Nr. 1638/2006 des Europäischen Parlaments und des Rates vom 24. Oktober 2006 zur Festlegung allgemeiner Bestimmungen zur Schaffung eines Europäischen Nachbarschafts- und Partnerschaftsinstruments, in: Amtsblatt der EU, Nr. L 310/2006, S. 1-14.

2 Europäische Kommission: Mitteilung an den Rat und das Europäische Parlament über die Stärkung der Europäischen Nachbarschaftspolitik, KOM (2006) 726 endg. 
nern, vor allem in den Sektoren Institutionenaufbau, Energie, Umwelt, Verkehr und Justiz/ Inneres. Hier geht es insbesondere um die synergetische Vernetzung bestehender Initiativen.

Unter der deutschen Ratspräsidentschaft konnte die Europäische Nachbarschaftspolitik auch in diesen Einzelbereichen inhaltlich signifikant vertieft werden. Dabei konnte auf die in vielen Bereichen bereits bestehende Zusammenarbeit aufgebaut werden. Neben der Schaffung weiterer Finanzierungsinstrumente möchte ich dabei die wirtschaftliche Zusammenarbeit, den interkulturellen Dialog, damit zusammenhängend den Gesamtansatz Migration, den Bereich Energie sowie die regionale Zusammenarbeit im Schwarzmeerraum hervorheben.

Schaffung weiterer Finanzierungsinstrumente: Mit der neu eingerichteten GovernanceFazilität sollen Partner, die besonders ,reformfreudig ' und erfolgreich bei der Umsetzung der Aktionspläne waren, für ihre Bemühungen prämiert werden. Hierfür sind für den Zeitraum 2007-2013 insgesamt circa 350 Millionen Euro vorgesehen.

Daneben tritt mit der neuen Nachbarschaftsinvestitionsfazilität (NIF) ein weiteres Instrument hinzu. Hiermit sollen vor allem Darlehen europäischer Finanzinstitutionen und nationaler Entwicklungsbanken unterstützt werden, die diese in den Partnerländern im Einklang mit den Prioritäten der Europäischen Union, insbesondere aber auch für Einzelprojekte in den Bereichen Energie, Infrastruktur und Umwelt, vergeben. Ziel ist es, die Wirkung des ENPI-Finanzinstruments zu maximieren und so größtmögliche Reformimpulse in den Partnerländern auszulösen. Für die Periode 2007-2013 soll die Fazilität mit 700 Millionen Euro aus Mitteln des ENPI gespeist werden.

Zusätzlich sollen bilaterale Beiträge der Mitgliedstaaten zur Maximierung des Hebeleffekts eingebracht werden. Die Kommission hatte eine solche Fazilität (ursprünglich als Fonds gedacht) in der Mitteilung vom 4. Dezember 2006 vorgeschlagen. Die Schlussfolgerungen des RAA vom Juni sowie der Fortschrittsbericht der Präsidentschaft unterstreichen die zukünftige Rolle dieser Fazilität. Wir unterstützen die Kommission bei der Einrichtung und inhaltlichen Ausgestaltung der Nachbarschaftsinvestitionsfazilität. Sie wird die Wirkung der budgetären Hebel der Europäischen Union verbessern und die Zusammenlegung der Ressourcen der wichtigsten Geber fördern.

Stärkung der wirtschaftlichen Integration: Ein zentraler Baustein der ENP ist eine stärkere wirtschaftliche Vernetzung. Ein verbesserter Zugang unserer Partner zum Binnenmarkt ist einer der größten Anreize der ENP. Die Europäische Union öffnet ihren Markt für die Partner. Dies ist auch in einer gezielt asymmetrischen Form möglich. Deren ,Kosten` für die Europäische Union würden durch den positiven, wirtschaftlichen wie politischen Nutzen in den Nachbarstaaten und damit in der Europäischen Union selbst weit aufgewogen. Auch die europäischen Verbraucher profitieren unmittelbar von einer solchen Marktöffnung. Ein besserer Marktzugang für die ENP-Partner ist nicht nur für die wirtschaftliche Situation der in den Partnerländern lebenden Menschen selbst, sondern auch für die Glaubwürdigkeit der Europäischen Union von erheblicher Bedeutung. Dies gilt insbesondere für die Sektoren, in denen sie komparative Vorteile haben. Umgekehrt müssen aber auch die ENP-Partner ihre Märkte nach und nach öffnen und die EU-Standards in handelsrelevanten Bereichen übernehmen.

In diesem Sinne gilt es vor allem, die Verhandlungen mit der Ukraine über ein ,vertieftes Abkommen' zügig fortzusetzen. Dabei handelt es sich um ein Abkommen zwischen der Europäischen Union und der Ukraine in Nachfolge des 2008 auslaufenden Partnerschafts- und Kooperationsabkommens. Mehrere Verhandlungsrunden haben bereits unter der deutschen Ratspräsidentschaft stattgefunden. Nach dem Beitritt der Ukraine zur WTO können auch die Verhandlungen über das im Rahmen des vertieften Abkommens vorgesehene Freihandels- 
abkommen aufgenommen werden. Der erfolgreiche Abschluss dieses ,Vorzeigeprojekts“ hätte auch Modell- und Signalwirkung für die anderen Partner. Dies gilt - spiegelbildlich für den Süden - auch für ein ,Statut avancé' für Marokko. Hierzu wurden unter deutscher Präsidentschaft die Arbeiten aufgenommen. Ein ,vertieftes Abkommen' im Rahmen der ENP könnte ein wichtiges Signal für die südlichen Partner sein, dass sich Reformen lohnen. Beide Länder, die auch als erste in den Genuss der Mittel aus der neu geschaffenen GovernanceFazilität kommen, wären damit Musterbeispiele für eine inhaltlich intensivierte ENP.

Bei weiteren Partnerländern sollten wir mit der Umsetzung der ENP zeitnah Fortschritte erzielen. So sollten der Republik Moldau zunächst autonome Zollpräferenzen gewährt werden. Ferner werden zur Zeit Machbarkeitsstudien über Freihandelsabkommen mit Armenien und Georgien erstellt.

Stärkung des Dialogs zu interkulturellem Austausch und Migration: Die Europäische Nachbarschaftspolitik soll in die Bevölkerung hineinwirken. Wichtig ist, dass die Bürger in den Mitgliedstaaten wie auch in den Partnerländern - ganz konkret im täglichen Leben von den Vorteilen der ENP profitieren. Hierbei spielt die Stärkung des interkulturellen Austausches durch die Förderung der Mobilität zwischen den EU-Mitgliedstaaten und den ENPLändern eine wichtige Rolle. Dem dienen zahlreiche Visaerleichterungsabkommen zwischen der Europäischen Union und den Ländern im Osten beziehungsweise Südosten der Europäischen Union (unter anderem mit den ENP-Partnern Ukraine und Moldau). Die Europäische Union hat ferner beschlossen, für bestimmte Personengruppen aus den östlichen Nachbarstaaten, die an ENP-Veranstaltungen teilnehmen, Visaerleichterungen vorzunehmen. Dies baut auf Regelungen auf, die seit 2003 für Bürger aus den südlichen Partnerstaaten gelten. Die Erleichterung der Mobilität trägt wesentlich zur Stärkung des unverzichtbaren interkulturellen Dialogs bei.

Vor allem den jungen Generationen sollte ein intensiver Austausch ermöglicht werden. Ausbildungsmaßnahmen wie zum Beispiel neue Stipendien im Rahmen des Erasmus-Mundus-Programms sollten daher weiter ausgeweitet werden.

Gleichzeitig liegt es im gemeinsamen Interesse, eine verbesserte Steuerung der Migration zu erreichen. Hierbei geht es um den gemeinsamen Schutz der Grenzen, die Eindämmung der illegalen Migration und die Bekämpfung von Menschenhandel und Schleuserkriminalität, zum Beispiel durch die Beteiligung der ENP-Länder an Operationen der Europäischen Grenzschutzagentur Frontex.

Das Phänomen Migration umfassend zu betrachten, das heißt auf Basis eines partnerschaftlichen Dialogs mit Herkunfts- und Transitländern einerseits die positiven - zum Beispiel entwicklungsfördernden - Aspekte der Migration zu nutzen und andererseits die negativen zu verringern und gezielt Armut zu bekämpfen, um an den Ursachen der Migration anzusetzen, ist die Grundidee des sogenannten Gesamtansatzes Migration, den die Europäische Union im Dezember 2005 verabschiedet hat. Unter deutscher Präsidentschaft wurde diese Strategie weiter fortentwickelt: Zum einen soll das Engagement für Afrika und den Mittelmeerraum verstärkt werden, zum anderen werden durch die Verabschiedung eines konkreten Aktionsplans für die östlichen und südöstlichen EU-Nachbarregionen nunmehr im Sinne einer geographischen Ausgewogenheit auch die dortigen ENP-Partnerländer in den Blick genommen.

Hervorzuheben ist insoweit das innovative Konzept der sogenannten Mobilitätspartnerschaften, das - sofern der Europäische Rat im Dezember 2007 entsprechend entscheidet - in Pilotpartnerschaften mit zwei bis drei Drittländern getestet werden soll. Solche Partnerschaften sollen einen Beitrag zu einer kohärenten Migrationspolitik leisten, indem - unter Beachtung der nationalen Zuständigkeiten und der spezifischen Bedürfnisse der Arbeitsmärkte - 
Maßnahmen zur Nutzung der Vorteile gut geregelter, legaler Migration mit Maßnahmen zur Eindämmung der illegalen Migration, zum Schutz von Flüchtlingen und zur gezielten Armutsbekämpfung kombiniert werden.

Energiebereich: Als sektorale Form der Zusammenarbeit ferner hervorzuheben ist der Bereich der Energie. Viele Nachbarstaaten der Europäischen Union liefern Erdgas und Erdöl oder fungieren als wichtige Transitstaaten. Zudem besteht in vielen Ländern ein großes Potenzial für den Einsatz erneuerbarer Energien wie Solar- und Windenergie oder Biomasse. Im Rahmen der ENP wird daher die Zusammenarbeit im Energiebereich stetig vertieft. Im Vordergrund stehen dabei die Harmonisierung der rechtlichen Rahmenbedingungen zur Erleichterung von Investitionen und des Handels, der Austausch und die Unterstützung bei Energieeffizienz, erneuerbaren Energien und Energietechnologien und die Verständigung über energiepolitische Fragen. Als Instrumente können spezielle Vereinbarungen wie mit der Ukraine, multilaterale Foren und dazugehörige Arbeitsgruppen oder konkrete Projekte und Vorhaben dienen.

Besonders weitgehend ist die geplante Ausdehnung der für die Staaten Südosteuropas mit EU-Beitrittsperspektive konzipierten Energiegemeinschaft auf die Ukraine und die Republik Moldau, die dort bereits Beobachterstatus haben. Die Mitglieder dieser Gemeinschaft übernehmen das EU-Recht in den Bereichen Strom und Gas. So entsteht mittelfristig ein großer gemeinsamer Markt für Strom und Gas. Mit der Ukraine ist vereinbart, dass sie die aus der Governance-Fazilität zur Verfügung stehenden Mittel für das Jahr 2007 (22 Millionen Euro) unter anderem als Budgethilfe zur Unterstützung der Infrastruktur beziehungsweise als Hilfe bei der Umsetzung der Ukraine-Energiestrategie und des Energiekapitels im Aktionsplan verwendet.

Regionale Zusammenarbeit im Schwarzmeerraum: Deutschland hat im Vorfeld und während der deutschen EU-Präsidentschaft die Entwicklung einer intensivierten EU-Politik gegenüber der Schwarzmeerregion angestoßen, mit dem Ziel, regionale Zusammenarbeit im Schwarzmeerraum zu fördern und die Beziehungen der Region mit der Europäischen Union auf allen Ebenen auszubauen. Der Schwarzmeerraum ist nicht nur wegen seiner beträchtlichen Krisenanfälligkeit von strategischer Bedeutung für die Europäische Union, sondern auch wegen seines großen wirtschaftlichen Potenzials. Mit dem EU-Beitritt Rumäniens und Bulgariens ist die Europäische Union zum Schwarzmeeranrainer geworden. Damit steigt das unmittelbare Interesse der Europäischen Union an Modernisierung und Stabilität der Schwarzmeerregion. Die Ratsschlussfolgerungen vom 14. Mai 2007 sowie der vom RAA am 18. Juni 2007 und Europäischen Rat am 21./22. Juni 2007 indossierte Präsidentschaftsbericht zur Weiterentwicklung der ENP sind Ausdruck des EU-Konsenses über die strategische Bedeutung des Schwarzmeerraums.

Die Schwarzmeersynergie-Initiative bezweckt vor allem eine praktische und ergebnisorientierte Zusammenarbeit in Bereichen mit grenzüberschreitender Bedeutung, in denen durch eine verbesserte regionale Kooperation Synergien geschaffen und Ressourcen effizienter genutzt werden können. Eine solche regionale Komponente ergänzt die bilateralen Maßnahmen der Europäischen Union für die Länder der Region. Die Kernbereiche für diesen regionalen Ansatz sind unter anderem Energie, Umwelt, Verkehr/Transport, Migrationsfragen, Bekämpfung der organisierten Kriminalität sowie Zusammenarbeit auf der Ebene der Zivilgesellschaft. Hier decken sich die Interessen der Europäischen Union und der Länder der Region.

Eine verstärkte regionale Zusammenarbeit im Schwarzmeerraum bringt nicht nur einen wirtschaftlichen Nutzen mit sich. Sie kann auch zur Vertrauensbildung und zum Abbau noch bestehender bilateraler Spannungen beitragen. 
Es sollen mit dieser Initiative keine neuen Institutionen geschaffen werden, sondern es geht in erster Linie darum, an schon bestehende Programme der Europäischen Union beziehungsweise Aktivitäten der Länder der Region anzuknüpfen und diese weiter zu verstärken.

Bereits unter slowenischer Präsidentschaft im ersten Halbjahr 2008 findet eine Überprüfung der bis dahin erfolgten Fortschritte bei der Umsetzung der Schwarzmeersynergie-Initiative statt. Es ist daher wichtig, dass die von der deutschen Präsidentschaft sowie der Kommission gemachten Vorschläge zur Vertiefung der regionalen Zusammenarbeit in den verschiedenen Kooperationsbereichen nun rasch konkretisiert und zügig umgesetzt werden.

\section{Fazit}

Die Europäische Union hat unter deutschem Vorsitz im 1. Halbjahr 2007 wichtige Schritte unternommen, um ihr Angebot an die ENP-Partner greifbarer und glaubwürdiger zu machen. Es geht nun um die Umsetzung dieses Angebots in konkrete Maßnahmen. Sofern es uns gelingt, unsere Transformationskraft gezielt einzusetzen, werden beide Seiten, Europäische Union und ENP-Partnerländer, von der Europäischen Nachbarschaftspolitik erheblich profitieren können. Dabei geht es nicht nur um die Fortschritte in den Einzelbereichen. Ziel ist es, neben einem einheitlichen Auftreten der Europäischen Union auch zu einer kohärenteren Wertegemeinschaft mit den ENP-Partnerländern zu gelangen. So haben wir beispielsweise beschlossen, den südkaukasischen Ländern in einzelnen Fällen die Möglichkeit zu geben, sich GASP-Erklärungen, Demarchen und Stellungnahmen der Europäischen Union anzuschließen. Das Interesse an einer solchen Wertegemeinschaft ist auf beiden Seiten vorhanden. Das machte unter anderem die breite hochrangige Präsenz auf Seiten der EUMitgliedstaaten und der ENP-Partnerländer auf einer Veranstaltung der Kommission zur Europäischen Nachbarschaftspolitik Anfang September, auf der ich die Bundesregierung vertrat, deutlich. Die ENP hat sich als zentrales Instrument der Gestaltung der EU-Beziehungen zu den Nachbarstaaten etabliert. Sie wird auch mittel- und langfristig unersetzbar sein, um Trennlinien abzubauen und der Dichotomie von ,EU-Beitritt: Ja oder Nein“ ihre Dramatik zu nehmen.

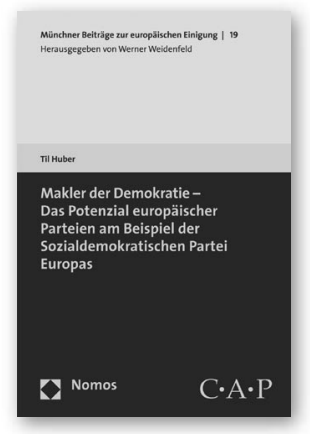

Makler der Demokratie -

Das Potenzial europäischer Parteien am Beispiel der Sozialdemokratischen Partei

\section{Europas}

Von Til Huber

2007, 124 S., brosch., 19,- €, ISBN 978-3-8329-2800-1

(Münchner Beiträge zur europäischen Einigung, Bd. 19)

Bitte bestellen Sie bei Ihrer Buchhandlung oder bei Nomos | Telefon 07221/2104-37 | Fax -43 www.nomos.de | sabine.horn@nomos.de

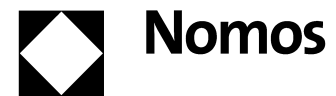

